\title{
Pathway-Specific Properties of AMPA and NMDA-Mediated Transmission in CA1 Hippocampal Pyramidal Cells
}

\author{
Nonna A. Otmakhova, Nikolai Otmakhov, John E. Lisman \\ Department of Biology and Volen Center for Complex Systems, Brandeis University, Waltham, Massachusetts 02454
}

CA1 pyramidal cells receive glutamatergic input from the entorhinal cortex through the perforant path (PP) and from CA3 through Schaffer collaterals (SC). The PP input terminates in the stratum lacunosum moleculare $\sim 300 \mu \mathrm{m}$ from the cell body, whereas SC synapses have a more proximal location in the stratum radiatum. We compared the properties of AMPA- and NMDA-mediated transmission at these two inputs. The AMPAmediated components have linear voltage dependence in both inputs. The reversal potential in the PP is only slightly more positive than in the SC, indicating that distal membrane voltage could be effectively set. The NMDA-mediated responses in the two pathways, however, are very different. The PP exhibits inward rectification, as evidenced by very low outward currents. The rectification persists in the absence of extracellular $\mathrm{Mg}^{2+}$. It cannot be attributed to clamping problems, because large outward AMPA currents can be observed even when conditions are modified to have the AMPA currents kinetically match the NMDA currents. Thus, it appears that the PP NMDA channels have novel properties. A second difference between the PP and SC pathways is that the PP has a larger NMDA/AMPA charge ratio. This difference could be observed under many conditions, including block of all voltage-dependent conductances and elimination of the negative resistance of NMDA channels by removing extracellular $\mathrm{Mg}^{2+}$. The difference in ratio thus cannot be attributed to regenerative currents. The higher NMDA component of the distal PP synapses could help to make these synapses more powerful under depolarizing conditions.

Key words: AMPA; CA1; D890; inward rectification; NMDA; perforant path; $Q X-314$; regenerative process; Schaffer collaterals; voltage dependence; whole-cell patch clamp; ZD7288
Dendrites create sites for the convergence of large numbers $\left(>10^{5}\right)$ of synaptic inputs. The difference in distances of synapses from the cell body means that inputs with different locations will undergo different electrotonic attenuation as current flows toward the soma. There is increasing evidence that synaptic responses can be affected by voltage-dependent conductance (Stuart and Sakmann, 1995; Hoffman et al., 1997; Seamans et al., 1997; Magee, 1998; Andreasen and Lambert, 1999). Some of these $\left(I_{\mathrm{A}}\right.$ and $I_{\mathrm{h}}$ are more concentrated in distal dendrites (Hoffman et al., 1997; Magee, 1998) and may preferentially attenuate distally generated synaptic inputs. Conversely, it was shown recently that distal synapses have a higher AMPA conductance than proximal synapses (Magee and Cook, 2000) and that this helps to equalize the effectiveness of synapses.

There is also growing evidence for complex dendritic processes involving NMDA conductance. NMDA channels control the initiation of dendritic spikes (Golding and Spruston, 1998; Calton et al., 2000), which can have a secondary effect on the EPSP (Hausser and Stuart, 2001; Hausser et al., 2001). In distal basal dendrites of cortical cells, NMDA channels can themselves generate a spike-like event (Schiller et al., 2000). It has been sug-

Received Sept. 26, 2001; revised Nov. 8, 2001; accepted Nov. 9, 2001.

This work was supported by the W. M. Keck Foundation; by National Institutes of Health Grants 2 RO1 NS27337-12 and P50 MH60450-01A1; by Alzheimer's Association Grant RG3-96-015; by Alzheimer's Association grants to J.L.; and by a National Alliance for Research on Schizophrenia and Depression Young Investigator Award to N.O. We greatly appreciate the discussions and advice from Drs. Dan Johnston, Jeff Magee, Piotr Bregestovsky, Lyle Borg-Graham, Nail Burnashev, Gary Westbrook, and Adam Kepecs.

Correspondence should be addressed to Dr. John E. Lisman, Volen Center for Complex Systems, Brandeis University, 415 South Street, Waltham, MA 02454. E-mail: lisman@brandeis.edu.

Copyright (C) 2002 Society for Neuroscience $\quad 0270-6474 / 02 / 221199-09 \$ 15.00 / 0$ gested (Cook and Johnston, 1999) that NMDA channels could help to produce location independence of synapses by making synapses into "perfect current sources" (a decrease in AMPA current with depolarization is balanced by an increase in NMDA current).

Given the complexity of synaptic events in the dendrites, it was of interest to determine whether different pathways that produce inputs at different distances from the soma have different AMPA and NMDA properties. The pyramidal cells of the hippocampal CA1 region provide a particularly favorable preparation for studying location-specific inputs. The "direct" pathway from the entorhinal cortex is called the perforant path (PP) and terminates on the most distant CA1 dendrites in the stratum lacunosum moleculare. This pathway is a major source of specific sensory information for the hippocampus (Vinogradova, 1984; McNaughton et al., 1989) and can evoke postsynaptic firing (Bragin and Otmakhov, 1979; Doller and Weight, 1982; Yeckel and Berger, 1990). A second input to CA1 is through the Schaffer collateral (SC) axons of CA3 cells that terminate more proximally in the stratum radiatum. Previous work has established that both of these inputs are glutamatergic. We used whole-cell recording to directly compare the properties of NMDA- and AMPA-mediated responses in the two inputs.

\section{MATERIALS AND METHODS}

Transverse hippocampal slices ( $350 \mu \mathrm{m}$ thick) were prepared from 17 - to 25-d-old Long-Evans rats. Part of the dentate gyrus and the CA3 field were cut from the slices by a single diagonal cut as described previously (Otmakhova and Lisman, 1999). Slices were preincubated in an inverse interface chamber for $2-6 \mathrm{hr}$ before an experiment (Otmakhova et al., 2000). For recording, slices were placed on a glass bottom of the recording chamber and superfused with artificial CSF (ACSF) using a pump with a flow rate of $1.5-2.25 \mathrm{ml} / \mathrm{min}$. ACSF contained (in mM): $\mathrm{NaCl} 120$, 
$\mathrm{NaHCO}_{3} 26, \mathrm{NaH}_{2} \mathrm{PO}_{4} 1, \mathrm{KCl} 2.5, \mathrm{MgSO}_{4} 1.3, \mathrm{CaCl}_{2} 2.5$, and D-glucose 20. In addition, $50 \mu \mathrm{M}$ picrotoxin (PTX) was used to block $\mathrm{GABA}_{\mathrm{A}}$ inhibition in most experiments. This is referred to as "standard ACSF" in the text. When needed, $100 \mu \mathrm{M} \pm \mathrm{APV}$ (NMDA blocker) or $10 \mu \mathrm{M}$ 1,2,3,4-tetrahydro-6-nitro-2,3-dioxo-benzo[f]quinoxaline-7-sulfonamide disodium (NBQX) (AMPA antagonist) was added to ACSF. For some experiments, the $\mathrm{MgSO}_{4}$ concentration in ACSF was decreased to 0 or $0.1 \mathrm{~mm}$ (low- $\mathrm{Mg}^{2+} \mathrm{ACSF}$ ). Before entry into the recording chamber, ACSF was saturated with a gas mixture of $95 \%$ oxygen and $5 \%$ carbon dioxide. All experiments were done at room temperature $\left(20-22^{\circ} \mathrm{C}\right)$.

Whole-cell patch clamp. Whole-cell voltage-clamp recordings were performed using an Axopatch-1D amplifier (Axon Instruments, Foster City, CA) with a low-pass filter set at $1 \mathrm{kHz}$ as described previously (Otmakhova et al., 2000). The patch pipettes had a resistance of 2.5-3.5 $\mathrm{M} \Omega$ when filled with pipette solution. For voltage clamp, the pipette solution contained (in mM): Cs-methanesulfonate 120, CsCl 20, HEPES 10, Mg-ATP 4, $\mathrm{Na}_{3}$-GTP 0.3, EGTA 0.2, Na-phosphocreatine 10, and QX-314 5, pH 7.3; osmolarity was 300 mOsm. In some experiments, $1 \mathrm{~mm}$ D890 was included to block voltage-gated $\mathrm{Ca}^{2+}$ channels. Patching was performed under visual control using infrared differential interference contrast optics (Olympus, Hamburg, Germany) and a CCD video camera. Recordings were made from cell bodies in the CA1 pyramidal layer, $30-80 \mu \mathrm{m}$ beneath the slice surface. To stimulate the PP and SC inputs, two monopolar stimulating electrodes (glass pipettes filled with ACSF; resistance, $0.25-0.35 \mathrm{M} \Omega$ ) were positioned on the stratum lacunosum moleculare $(\sim 300 \pm 9 \mu \mathrm{m}$ from the cell body) and the upper third of stratum radiatum $(\sim 160 \pm 11 \mu \mathrm{m}$ from the cell body), respectively. Each input was stimulated every $10-20 \mathrm{sec}$ with a $2-50 \mu \mathrm{A}, 150 \mu$ s square pulse delivered through current isolation units (Isolator-11; Axon Instruments). The two inputs were stimulated alternately. In all cases, experiments started 30-45 min after the breaking of the seal to provide for sufficient diffusion time for intracellular channel inhibitors. Whole-cell currents in response to PP and SC stimulation were measured in voltageclamp mode at different holding potentials. Changes in holding potential were controlled manually. Each step lasted 2-4 $\min$ (to acquire 5-10 EPSCs), and voltage between steps was ramped at $\sim 2 \mathrm{mV} / \mathrm{sec}$. Series and input resistances were monitored after each response by measuring the peak and steady-state currents in response to $2-4 \mathrm{mV}, 38 \mathrm{msec}$ hyperpolarizing steps. Cells with unstable series resistance ( $>20 \%$ change) or cells with a series resistance of $>13 \mathrm{M} \Omega$ were discarded from further analysis. Recorded signals were digitized at $5-10 \mathrm{kHz}$ and then stored and analyzed using custom software written in Axobasic (Axon Instruments). For whole-cell current clamp, an Axoclamp-2A amplifier (Axon Instruments) was used. The pipette solution contained (in $\mathrm{mm}$ ): K-methylsulfate 150, KCl 20, HEPES 10, Mg-ATP 4, Na - GTP 0.3 , EGTA 0.1, Na-phosphocreatine 10, and QX-314 5, pH 7.3; osmolarity was $300 \mathrm{mOsm}$. Most of the chemicals for intracellular solutions and ACSF were purchased from Sigma (St. Louis, MO). Picrotoxin, \pm APV, NBQX, and QX-314 were purchased from Sigma-RBI (Natick, MA). D890 was obtained from Knoll (Ludwigshafen, Germany). ZD7288 was purchased from Tocris (Bristol, UK).

Statistical analysis. Five to ten evoked responses were averaged for each cell before measurement. For the measurements of response area, we used an absolute sum of recorded currents over time starting immediately after the stimulus artifact and until the end of the recorded trace (425 msec). The peak amplitude $\left(A_{\text {max }}\right)$ was determined in a $3 \mathrm{msec}$ window centered at the peak of the synaptic response. The area or peak current of a synaptic response was calculated by subtracting the average value of the baseline data points in a $15 \mathrm{msec}$ window before the stimulus. Kinetic variables of average (10 traces) AMPA EPSC were measured at the holding potential of $-65 \mathrm{mV}$. Peak latency $\left(T_{\max }\right)$ was measured from the end of the stimulus artifact to the peak of EPSC. Rise time was estimated between 10 and $90 \%$ of points on the rising slope (rise time), and decay time constants $(\tau)$ were calculated by exponential (first order) fitting in the Microcal Origin program package (Microcal Inc., Northampton, MA). Data were averaged between cells, and means and SEMs were calculated. A two-tailed $t$ test or a one-factor ANOVA with $\alpha<0.05$ was used for statistical analysis (Microsoft Excel, Seattle, WA). All comparisons between the SC and PP inputs were done after normalization of results in each input. Voltage characteristics of isolated AMPA EPSCs were linearly fit in Microcal Origin using averaged data of 10 cells (means) and SEMs as a weight. We did not correct for junction potential.

\section{RESULTS}

PP synapses are located at the distal region of the apical dendrites $\sim 300 \mu \mathrm{m}$ from the cell body. The dendrites in this region are too thin to be patched directly. Therefore, to study the PP path responses, we recorded responses in the cell body, but we took measures to improve the voltage control of distal dendrites. The intracellular solution contained $\mathrm{Cs}^{+}$to block most $\mathrm{K}^{+}$channels (Hestrin et al., 1990) and QX-314 to block Na ${ }^{+}$channels (Connors and Prince, 1982), $I_{\mathrm{h}}$ channels (Perkins and Wong, 1995), G-protein-coupled $\mathrm{K}^{+}\left(\mathrm{GABA}_{\mathrm{B}}\right)$ channels (Lambert and Wilson, 1993), and $\mathrm{Ca}^{2+}$ currents (partially) (Talbot and Sayer, 1996). In some experiments, we also included $1 \mathrm{~mm}$ D890 to produce a more complete block of $\mathrm{Ca}^{2+}$ currents (Kovalchuk et al., 2000). After the whole-cell configuration had been obtained, measurements were delayed for 30-45 min to ensure that these blockers had time to reach distal dendrites. $\mathrm{GABA}_{\mathrm{A}}$ and glycine chloride channels (Yoon et al., 1998) were blocked by $50 \mu \mathrm{M}$ picrotoxin in the bath. Collectively, these measures increase membrane resistance and therefore enhance the accuracy with which distant synaptic responses can be measured (Carnevale and Johnston, 1982; Spruston et al., 1993). Because synaptic currents are distorted when series resistance is high (Major, 1993), we did not analyze records with a series resistance of $>13 \mathrm{M} \Omega$. Finally, we based our conclusions primarily on measurements of the area of the synaptic response rather than the peak. This is because electrotonic properties produce strong attenuation of the response peak with increasing dendritic distance (Carnevale and Johnston, 1982; Major, 1993; Major et al., 1993a,b; Spruston et al., 1993) but have comparatively little effect on the response integral (Carnevale and Johnston, 1982; Spruston et al., 1993). Calculations indicate that when clamp measurements are made at a distance of one length constant, peak current is attenuated by $80-90 \%$, whereas charge is decreased by only $20-30 \%$ (Spruston et al., 1993). According to some estimates, the full electrotonic length of the CA1 apical dendrite is $0.4-0.8$ of the length constant (Hestrin et al., 1990; Major et al., 1993a), especially in the in vitro situation, where background synaptic inputs are reduced (Bernander et al., 1991). Measuring EPSC area (charge) is thus the most accurate method available for characterizing the PP synapses.

\section{Characteristics of the AMPA-mediated response at SC and PP inputs}

We first compared the properties of the AMPA-mediated responses in the PP and SC pathways. Figure 1 shows averaged (10 cells) data on the area of the AMPA-mediated EPSC (100 $\mu \mathrm{M}$ \pm APV) as a function of voltage for the PP and SC inputs. The voltage dependence was linear in both cases $(r=0.99 ; p<0.001)$. The reversal potential was $6.8 \pm 2.8 \mathrm{mV}$ in the SC input and $13.3 \pm 2.6 \mathrm{mV}$ in the PP input, which is significantly higher $(p<$ $0.05 ; n=10)$. A linear current-voltage curve is expected for AMPA channels that contain glutamate receptor B (GluR-B) subunit (Verdoorn et al., 1991). Examples of the SC and PP EPSC at -65 and $+40 \mathrm{mV}$ are shown in Figure 1, $C$ and $D$, and the general characteristics of an EPSC at $-65 \mathrm{mV}$ are given in Table 1. All the kinetic characteristics in the PP (Fig. 1D, Table 1) were slower than in the SC input (Fig. $1 C$, Table 1), as expected given its more distal location. The rise and decay characteristics of the SC input in our study were comparable with those already described for SC inputs (Hestrin et al., 1990) and for similarly distanced commissural/associational inputs to CA3 pyramidal cells (Williams and Johnston, 1991). 

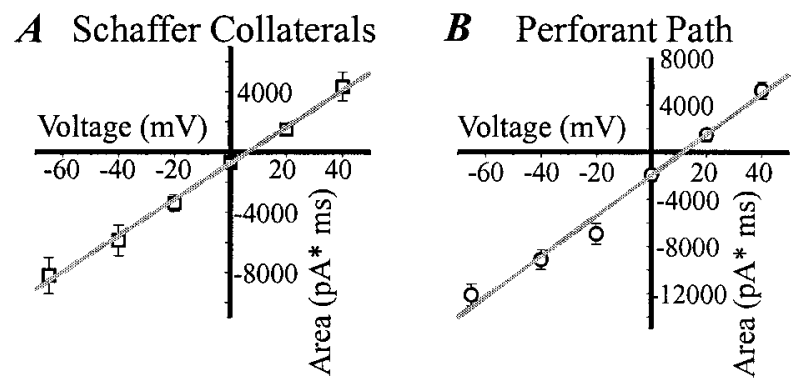

\section{$C$ Schaffer Collaterals}

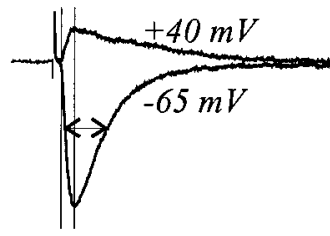

$D$ Perforant Path
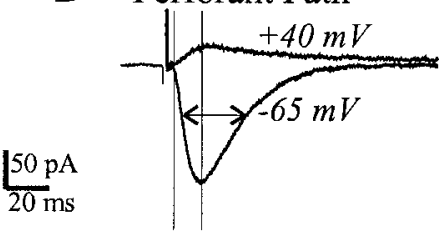

Figure 1. Similar current-voltage curves for isolated AMPA EPSCs in the SC and PP inputs. $A$, Voltage dependence (the average of 10 cells) of the SC EPSC area and a linear fit (gray line) using SEM as a weight $(r=$ $0.99 ; p<0.001)$. $B$, Voltage dependence $(10$ cells $)$ of the PP EPSC area and a linear fit (gray line; $r=0.99 ; p<0.001)$. $C$, The average (10 traces) SC EPSC at -65 and $+40 \mathrm{mV}$. $D$, The average $(n=10)$ PP EPSC from the same cell. Vertical lines mark the rising phase of EPSC; horizontal arrows mark the half-width. Calibration is shown in the middle.

\section{Table 1. Characteristics of AMPA EPSC in the PP and SC inputs at $-65 \mathrm{mV}(n=10)$}

\begin{tabular}{lcc} 
& $\mathrm{SD}$ & $\mathrm{PP}$ \\
\hline Amplitude, pA $\left(A_{\max }\right)$ & $156.3 \pm 20$ & $160.3 \pm 11$ \\
Area, $\mathrm{pA} \times$ msec & $8208 \pm 1211$ & $12135 \pm 927$ \\
Latency to peak, msec $\left(T_{\max }\right)$ & $8.6 \pm 0.4$ & $14.0 \pm 1.3$ \\
Rise time, msec $(10-90 \%$ & & \\
$\left.\quad A_{\max }\right)$ & $3.1 \pm 0.2$ & $6.0 \pm 0.66$ \\
Decay time constant, msec $(\tau)$ & $12.36 \pm 1.05$ & $17.72 \pm 1.26$
\end{tabular}

Except for the amplitude, all differences between the inputs are statistically significant. Note that these are pairwise comparisons using a paired $t$ test (two-tailed). These are less sensitive to variability between the cells.

\section{Characteristics of the NMDA-mediated response at SC and PP inputs}

We subsequently characterized the NMDA response in the two pathways. At the start of the experiments, the strength of synaptic stimulation was adjusted so that the response areas were approximately equal in the two pathways at $-60 \mathrm{mV}$, where the response is primarily attributable to the AMPA channels. A $10 \mu \mathrm{M}$ concentration of NBQX was then added to isolate the NMDA component. The current-voltage curve of the NMDA component for the two pathways is shown in Figure $2 A$. In the -20 to -70 $\mathrm{mV}$ range, both pathways exhibit the decline of response with hyperpolarization (negative slope) that is characteristic of NMDA channels. Both pathways exhibited a maximal inward current at $-20 \mathrm{mV}$. The curves normalized by the maximal inward current (Fig. 2B), however, show a difference between pathways at positive membrane potentials: the outward currents in the PP are much smaller than in the SC. Figure $2 C$ shows the examples of outward current at +60 scaled by maximal inward current at $-20 \mathrm{mV}$. The ratio of the area of the NMDA-mediated EPSC at +60 to that at $-20 \mathrm{mV}$ was $3.2 \pm 0.4$ for the SC but only $0.56 \pm 0.2$ for the PP $(p<0.001 ; n=10)$. This means that at the $\mathrm{PP}$, outward current at +60 is approximately six times smaller

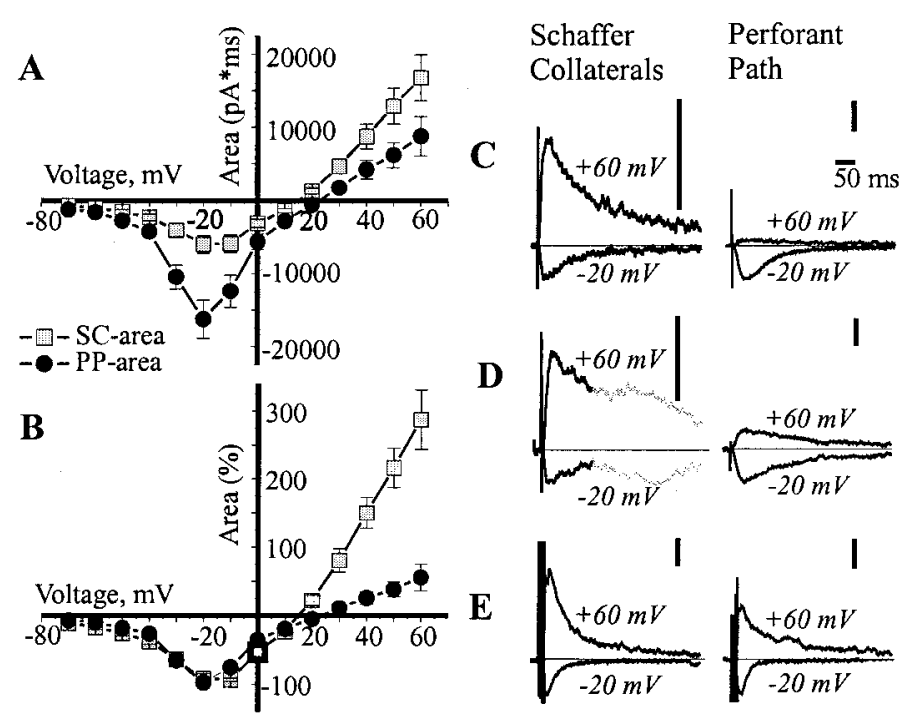

Figure 2. Different voltage dependence of the isolated NMDA response in PP and SC inputs. $A$, Voltage dependence of the isolated NMDA EPSC area in the SC and PP inputs (average of 10 cells). $B$, The same data normalized by a maximal inward current in each cell. $C$, The SC and PP NMDA EPSC (average of 5 traces) at two voltages $(-20$ and $+60 \mathrm{mV})$ in standard ACSF. D, The SC and PP NMDA EPSC (average of 5 traces) at -20 and $+60 \mathrm{mV}$ in ACSF without $\mathrm{Mg}^{2+}$. The early component of the $\mathrm{SC}$ response is drawn in black; the late polysynaptic component is marked in gray. E, The summated SC and PP AMPA EPSC to four stimuli at 200 $\mathrm{Hz}$ (average of 5 traces, artifact is truncated) at -20 and $+60 \mathrm{mV}$ in standard ACSF containing $100 \mu \mathrm{M} \pm \mathrm{APV}$ and $200 \mu \mathrm{M}$ cyclothiazide. For easier visualization, the EPSC traces in $C-E$ were scaled by the peak currents at $-20 \mathrm{mV}$. The individual calibration bars $(50 \mathrm{pA})$ are shown to the right of each pair of traces; the common time scale is $50 \mathrm{msec}(C$, top right).

than expected on the basis of the SC response. We checked whether the $+60 /-20$ ratio depended on the magnitude of the maximal inward charge. We found that in both inputs, there was no correlation between the size of the EPSC at -20 and the $+60 /-20$ ratio $(r=0.49, p>0.15$ in SC input; $r=0.09, p>0.8$ in PP input; $n=10)$. The ability of the PP to pass inward current much better than outward current will be called "inward rectification."

We were concerned that some other current might be contaminating the outward synaptic responses. When we checked this by applying an NMDA antagonist ( \pm APV, $100 \mu \mathrm{M})$ in addition to 10 $\mu \mathrm{M}$ NBQX, however, we found that the entire synaptic response at $+60 \mathrm{mV}$ was blocked $(n=3)$. We also checked whether the inward rectification might occur because of slow inactivation produced by the long depolarizations that we generally used. If faster depolarizations were used $(n=2)$, however, the rectification was still present.

Figure 1 demonstrates that the AMPA-mediated outward currents in the PP appear normal, and Figure $2 A-C$ shows the surprisingly small NMDA-mediated outward current, but these observations were made in different cells. In Figure 3, these differences are demonstrated in the same cell by the dissection of the NMDA and AMPA currents with $100 \mu \mathrm{M} \pm \mathrm{APV}(n=2$; Fig. $3 A)$ or $10 \mu \mathrm{M} \operatorname{NBQX}(n=3$; Fig. $3 B)$.

Because the NMDA conductance has a negative slope, there was a possibility that the NMDA-dependent regenerative process could have contributed to the EPSC area at $-20 \mathrm{mV}$ (and therefore change the $+60 /-20$ ratio). To control for this, we repeated our measurements after 30-40 min of perfusion with 
$\underline{\text { Schaffer Collaterals }}$
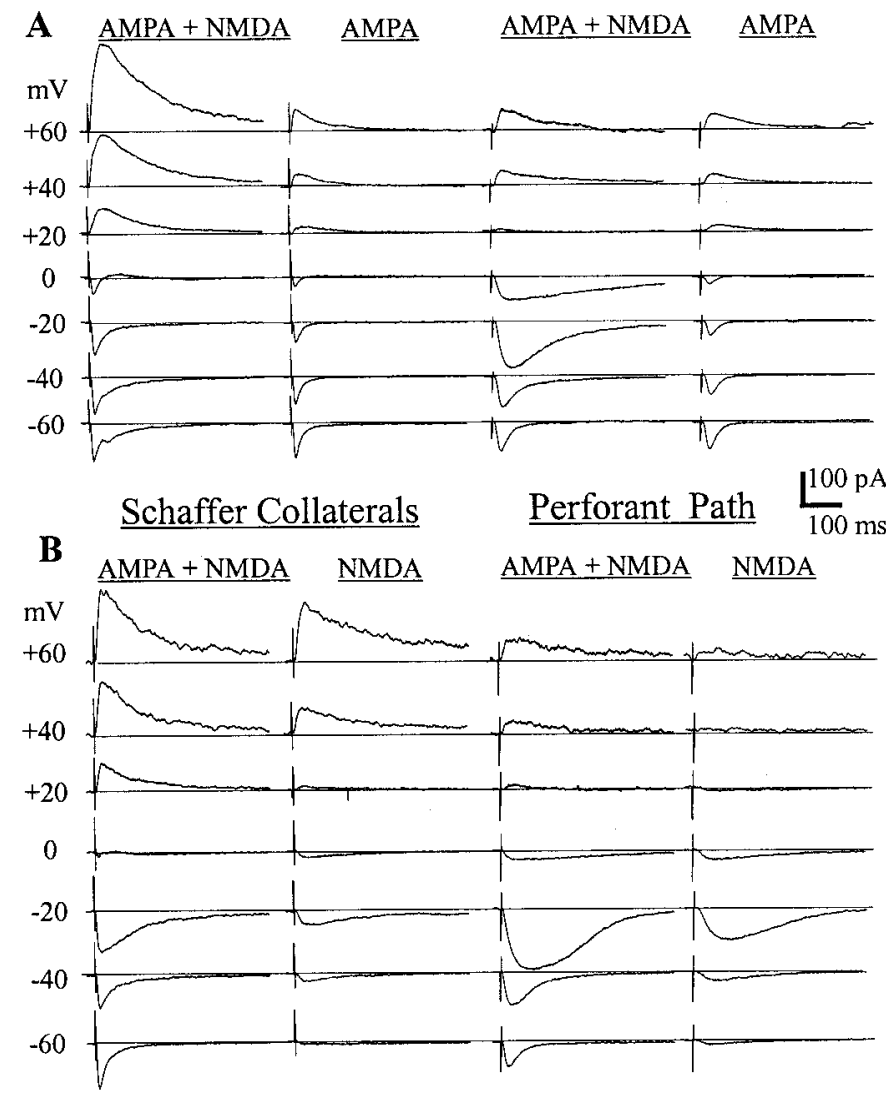

Figure 3. Examples of voltage dependence of the EPSC and its AMPA or NMDA component in the SC and PP inputs. $A$, The mixed EPSC ( first and third columns) and the isolated AMPA EPSC (second and fourth columns) in the SC and PP inputs in the same cell. At $-60 \mathrm{mV}$, the major fraction of the SC and PP EPSC area is attributable to the AMPA conductance. At $+60 \mathrm{mV}$, most of the SC EPSC area is attributable to the NMDA, whereas most of the PP EPSC area is still attributable to the AMPA conductance. The isolated AMPA EPSC behaves similarly in both inputs with fairly close values of reversal potential. $B$, The mixed EPSC ( first and third columns) and the isolated NMDA EPSC (second and fourth columns) in the SC and PP inputs in a similar cell (different from $A$ ). At $-60 \mathrm{mV}$, the AMPA current determines EPSC area in both inputs. At $+60 \mathrm{mV}$, the AMPA blockade does not affect the SC EPSC area but strongly decreases the PP EPSC area. Only 7 of 14 voltage points are shown. Each trace is an average of five. Calibration bars are shown in the middle right.

ACSF without $\mathrm{Mg}^{2+}$. To avoid the possibility of voltagedependent $\mathrm{Ca}^{2+}$ channels contributing to the regenerative process, these channels were blocked by addition of D890 (1 mM in $\mathrm{Cs}^{+}$-based intracellular solution containing $5 \mathrm{~mm}$ QX-314). In control experiments, D890 blocked all the signs of the $\mathrm{Ca}^{2+}$ spike that are normally seen in response to fast depolarizations $(+65$ $\mathrm{mV} / 200 \mathrm{msec}$ ). Furthermore, $\mathrm{Ca}^{2+}$ imaging showed that after a 20-30 min application of D890, the spike-induced $\mathrm{Ca}^{2+}$ entry into distal dendrites was completely blocked (R. Conti and J. Lisman, unpublished observations). In ACSF without $\mathrm{Mg}^{2+}$, the negative slope of the NMDA-mediated response was eliminated, as expected. In the PP, however, the $+60 /-20$ ratio was essentially the same as in standard $\mathrm{Mg}^{2+} \operatorname{ACSF}(0.65 \pm 0.2 ; n=6 ; p>$ $0.6)$. Therefore, inward rectification of the NMDA-mediated EPSC in the PP persisted in the absence of extracellular $\mathrm{Mg}^{2+}$. In the SC input, it was impossible to avoid late polysynaptic EPSC components in the absence of $\mathrm{Mg}^{2+}$, which are thought to be caused by the connections between the CA1 pyramidal cells (Mlinar et al., 2001). Therefore, we measured both the early monosynaptic component (Fig. 2D, black) and the entire area of the SC EPSC. By both measures, the $+60 /-20$ ratio in the SC was significantly higher than in the PP input $(p<0.05 ; n=6), 1.79 \pm$ 0.5 when the entire EPSC area was integrated and $1.89 \pm 0.5$ when only the area of the early monosynaptic component was measured. The $+60 /-20$ ratio in the SC in the absence of $\mathrm{Mg}^{2+}$ was insignificantly smaller than that in standard $\mathrm{Mg}^{2+}(p<0.1)$.

The question remained as to whether the rectification in the PP might be a result of voltage escape affecting the distal NMDA current differently because of its slow time course. To answer this question, we performed experiments in which we artificially prolonged the duration of the isolated AMPA-mediated responses (in $100 \mu \mathrm{M} \pm \mathrm{APV}$ ). The prolongation was achieved by combining the summation of AMPA current to a burst of four stimuli at 200 $\mathrm{Hz}$ with the inhibition of desensitization of AMPA channels by $200 \mu \mathrm{M}$ cyclothiazide (Calton et al., 2000). As a result, the peak latency and the area of the EPSC to the burst were substantially prolonged (Fig. 2E), becoming comparable with the isolated NMDA current. However, we found that this prolonged AMPA EPSC showed no signs of rectification at positive membrane potentials $(n=5)$. Therefore, inward rectification in the PP was selective for the NMDA EPSC and was not a secondary result of the long duration of the EPSC.

\section{NMDA/AMPA ratio in the two inputs}

We subsequently examined whether the AMPA and NMDA components are in similar proportions at the SC and PP inputs. We started with the holding voltage of $-65 \mathrm{mV}$ in standard ACSF and adjusted the SC and PP EPSC to be of approximately the same size. The cell was then depolarized to $-20 \mathrm{mV}$, a voltage at which both pathways have maximal NMDA current (Fig. 2). At this voltage, the synaptic currents were measured in control solution and after a 5 min application of APV $(100 \mu \mathrm{M})$. Examples of EPSCs are shown in Figure 4A. The NMDA/AMPA ratio was determined as the control area minus APV area, all divided by APV area. The ratios for both the PP and SC were determined in each of 13 cells, and the results are plotted in Figure $4 C$. In both pathways, the ratio was highly variable across cells, as reported previously for the SC pathway (Spruston et al., 1995). Figure $4 C$ compares the NMDA/AMPA ratio in the two pathways. If the ratios were the same, the points would be on the solid line $(Y=X)$. Figure $4 C$ shows, however, that the points were consistently above the line, indicating that the NMDA/AMPA ratio in the PP $(8.6 \pm 0.8)$ was consistently higher than in the SC input $(5.3 \pm 0.9 ; p<0.05$; Fig. $4 C)$. There was only one cell in which the ratio was higher in the SC input (without this outlier, the NMDA/AMPA ratio was $8.8 \pm 0.9$ in the PP and $4.7 \pm 0.8$ in the SC; $p<0.01)$.

In interpreting these data, we need to consider the possibility that voltage escape in the distal subsynaptic region might bias the data. Both theoretical and experimental considerations are relevant here. Published models (Carnevale and Johnston, 1982; Major, 1993; Major et al., 1993a,b; Spruston et al., 1993) suggest that in the case of insufficient voltage control, our method would systematically underestimate the NMDA/AMPA ratio in the PP. This would happen because at $-20 \mathrm{mV}$, we first measured the large control (AMPA plus NMDA) EPSC and then the small residual AMPA EPSC. The modeling works state that voltage escape would suppress the larger response (AMPA plus NMDA) 

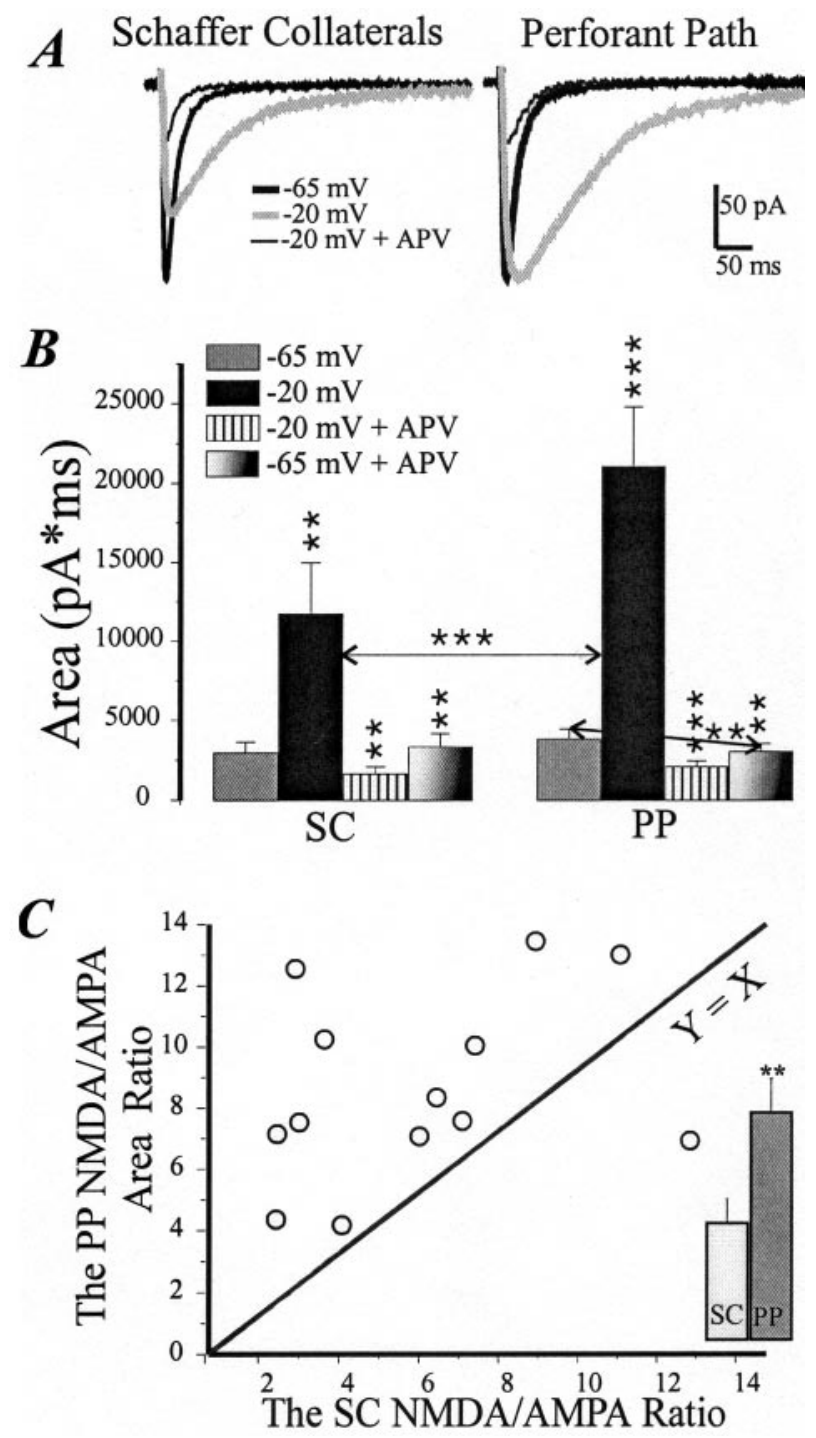

Figure 4. The NMDA/AMPA ratio at $-20 \mathrm{mV}$ in the PP is higher than in SC input. $A$, The PP and SC EPSC (average of 10 traces) in controls (at -65 and $-20 \mathrm{mV}$ ) and in the presence of APV. Calibration is shown in the bottom right corner. $B$, With depolarization, the PP EPSC area increased approximately twice as strongly as the SC EPSC area (average of 13 cells). The blockade of NMDA receptors decreases the area in both inputs to equal size. Asterisks indicate the significance of changes compared with previous conditions $\left({ }^{*} p<0.05 ;{ }^{*} p<0.01\right.$; $\left.{ }^{* * *} p<0.001\right)$. The horizontal arrow marks the significance of " -65 to $-20 \mathrm{mV}$ " and " -20 $\mathrm{mV}$ to $-20 \mathrm{mV}$ in APV" changes in paired $t$ test. The tilted arrow marks a significant decrease of the PP EPSC area at $-65 \mathrm{mV}$ after the blockade of NMDA receptors. $C$, A scatter diagram of the NMDA/AMPA area ratio in the PP plotted against the ratio in the SC for 13 cells. Each circle represents one cell. ${ }^{* *} p<0.01$. A $Y=X$ function plot is added for comparison. Inset, The bar graph shows the difference in averaged NMDA/AMPA ratios between the inputs.

more strongly than the smaller response (AMPA). Similarly, it would more strongly suppress slow (NMDA) responses than fast (AMPA) ones. Because of these properties, the NMDA component of response calculated by subtraction would appear smaller, and the NMDA/AMPA ratio would be underestimated.

There was also a possibility that a regenerative process contributed to measured responses and affected our calculations of NMDA/AMPA ratios. There are two special concerns. First, synaptic events in the distal inputs can be amplified by voltage- dependent $\mathrm{Na}^{+}$(Stuart and Sakmann, 1995; Andreasen and Lambert, 1999) or $\mathrm{Ca}^{2+}$ (Seamans et al., 1997) channels and attenuated by $I_{\mathrm{h}}$ (Magee, 1999) channels. In our experiments, the NMDA/AMPA differences should not be affected by these voltage-dependent channels, because they were strongly inhibited. Nevertheless, there was still a possibility that residual voltagegated $\mathrm{Ca}^{2+}$ channels might contribute more to the PP EPSC compared with the SC EPSC and thus bias NMDA/AMPA ratios. To determine whether the differences in the NMDA/AMPA ratio could be attributed to the voltage-dependent $\mathrm{Ca}^{2+}$ channels, we blocked these channels by intracellular application of the nonspecific blocker D890. The results of experiments with D890 are shown in Figure $5 A$ and are very similar to these without D890 (Fig. 4B). The difference in NMDA/AMPA ratios between the SC and PP $(3.7 \pm 0.8$ in the SC and $6.6 \pm 1.1$ in the PP; $p<$ 0.05) was comparable with what was found without D890. We conclude that this difference cannot be attributed to the action of voltage-dependent $\mathrm{Ca}^{2+}$ channels. Another possibility is that the differences could be attributable to $I_{\mathrm{h}}$ channels (Fig. $5 B$ ). To examine the role of this conductance, we used a $40 \mu \mathrm{M}$ concentration of the extracellular $I_{\mathrm{h}}$ inhibitor ZD7288 (Magee, 1999). The use of this bath-applied compound gives assurance that the effect is equal at all distances from the soma. As before, at -20 $\mathrm{mV}$, the NMDA/AMPA ratio in the SC $(5.8 \pm 1.1)$ was significantly smaller than in the PP input $(10.8 \pm 0.8 ; p<0.05 ; n=5)$.

The second concern was the possible role of the NMDAdependent regenerative process. In general, it could only cause the maximal inward NMDA current to occur at more hyperpolarized membrane potentials than expected under perfect voltage control. If so, the AMPA-dependent charge would be larger at a more hyperpolarized potential, causing underestimation of the NMDA/AMPA ratio in the PP. Despite these theoretical considerations, additional experimental controls seemed warranted. The NMDA-dependent regenerative process depends on the negative slope conductance produced by an $\mathrm{Mg}^{2+}$ block. Therefore, we measured the ratios after lowering the extracellular $\mathrm{Mg}^{2+}$ concentration. Initially we used a current-clamp method and $\mathrm{K}^{+}$-based intracellular solution (with QX-314) in the patch pipette (Fig. 6). After $10 \mathrm{~min}$ of perfusion with $0.1 \mathrm{mM} \mathrm{Mg}^{2+}$ and $50 \mu \mathrm{M}$ picrotoxin, the NMDA channels were blocked by $100 \mu \mathrm{M}$ \pm APV. To estimate the NMDA/AMPA ratios, we used the following formula: EPSP area in low $\mathrm{Mg}^{2+}$ plus PTX minus the area in low $\mathrm{Mg}^{2+}$ plus PTX plus APV, divided by the area in low $\mathrm{Mg}^{2+}$ plus PTX plus APV. Figure $6 C$ shows a scatterplot of PP versus $\mathrm{SC}$ ratios and averaged data (Fig. $6 C$, inset). It can be seen that the NMDA/AMPA ratio in the PP $(2.69 \pm 0.4)$ was nearly twice as large as in the SC $(1.46 \pm 0.3 ; p<0.01 ; n=11)$. In a second series of experiments, we measured the NMDA/AMPA ratio under voltage clamp (data not shown). We used a $\mathrm{Cs}^{+}$based intracellular solution containing $5 \mathrm{~mm} \mathrm{QX}-314$ and $1 \mathrm{~mm}$ D890 as in Figure $5 A$. Measurements were made at $-65 \mathrm{mV}$. Instead of blocking the NMDA channels, we blocked the AMPA channels with $10 \mu \mathrm{M}$ NBQX. The NMDA/AMPA ratio was calculated using the following formula: (EPSC area in low $\mathrm{Mg}^{2+}$ plus $\mathrm{NBQX}) /\left(\right.$ EPSC area in low $\mathrm{Mg}^{2+}$ minus the area in low $\mathrm{Mg}^{2+}$ plus NBQX). The voltage-clamp results were comparable with the current-clamp data. The NMDA/AMPA ratio in the PP $(2.2 \pm 0.5)$ was again approximately twice as high as in the SC input $(0.92 \pm 0.2 ; p<0.01 ; n=7)$. We conclude by these tests that the larger NMDA/AMPA ratio in the PP is not an artifact produced by a regenerative process. 
Figure 5. The greater NMDA component in the PP compared with the SC EPSC persists after the inhibition of voltage-dependent channels. Analyzed and marked as in Figure 4B. A, The intracellular $\mathrm{Ca}^{2+}$ channel blocker D890 (1 mM, 6 cells) does not decrease the differences between the two inputs that depend on NMDA current. $B$, In the presence of the extracellular $I_{\mathrm{h}}$ inhibitor ZD7288 (40 $\mu \mathrm{M}, 5$ cells), the changes in EPSC area that depend on NMDA current are still stronger in PP than in SC input. A $40 \mu \mathrm{M}$ concentration of ZD7288 inhibited both the SC and PP EPSC by $10-40 \%$. That is why the baseline EPSC area at $-65 \mathrm{mV}$ is smaller in these experiments than in previous experiments. We did not investigate the mechanism of this inhibition but performed our experiments $\sim 30$ min after the start of

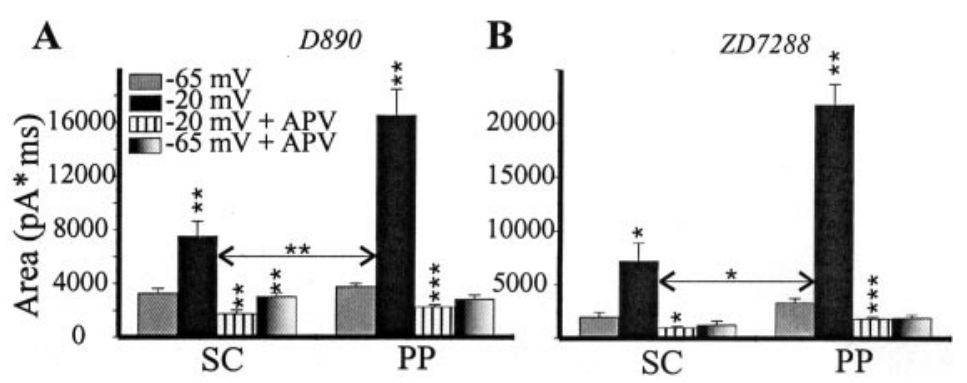
ZD7288 application to reach steady state. Asterisks indicate the significance of changes compared with previous conditions $\left({ }^{*} p<0.05 ;{ }^{* *} p<0.01 ;{ }^{* * *} p\right.$ $<0.001)$. The horizontal arrow marks the significance of " -65 to $-20 \mathrm{mV}$ " and " $-20 \mathrm{mV}$ to $-20 \mathrm{mV}$ in APV" changes in paired $t$ test.

There was a possibility that NMDA/AMPA ratio differences depended on the distance of the input from the cell body rather than on the origin of the input axons. After each experiment, we measured the distance between the stimulating and recording electrodes as an estimate of the distance of the stimulated synapses from the cell body. The data were used to determine whether there was a correlation between the NMDA/AMPA ratio and this distance. The correlations were insignificant in either input. In the SC, the coefficient of correlation was 0.35 in voltage clamp $(p>0.25 ; n=12)$ and -0.48 in current clamp $(p>0.12 ; n=11)$. In the PP, the coefficients were $0.13(p>0.66)$ and $0.22(p>0.5)$, respectively.

The voltage-clamp data in Figures 4 and 5 also address the question of whether presynaptic NMDA receptors could account for the NMDA/AMPA ratio differences between the two pathways. Figure $7 A$ shows the SC and PP NMDA/AMPA ratios at $-20 \mathrm{mV}$ under three experimental conditions. These data were obtained using pharmacological inhibition of NMDA channels by antagonist. If presynaptic NMDA receptors were present in only one input, the measurement of NMDA/AMPA ratios could be misleading. However, Figure $7 B$ shows how the ratios can be estimated without using the antagonist. At $-65 \mathrm{mV}$, the EPSC is primarily attributable to AMPA channels, and the increase in EPSC area with depolarization to $-20 \mathrm{mV}$ is attributable to the NMDA channels (AMPA current is decreased by depolarization). Figure $7 B$ shows that under all experimental conditions, the percentage of increase in EPSC area with depolarization in the $\mathrm{PP}$ is twice as large as in the SC input. Although there may be no presynaptic NMDA receptors in the CA1 region (Johnson et al., 1996), our results indicate that even if they were present, they could not account for the NMDA/AMPA ratio differences between the inputs.

As mentioned in the introductory remarks, one of the suggested roles for NMDA channels is to convert the synapse into a perfect current source (independent of membrane voltage) (Cook and Johnston, 1999). It was therefore of interest to see how different NMDA/AMPA ratios in the two inputs would affect the voltage dependence of the combined EPSC (containing AMPA and NMDA components). We measured the peak current and the total charge of combined EPSCs between -70 and $0 \mathrm{mV}$ in six cells. We found that the peak current in the PP was constant between -70 and $-40 \mathrm{mV}(F=0.09 ; p>0.95$ in a single-factor ANOVA) and then increased at $-20 \mathrm{mV}$ by $\sim 65 \%(p<0.01)$. In the SC, peak current decreased with depolarization. The total charge, on the contrary, was more constant in the SC: it did not change between -70 and $-40 \mathrm{mV}(F=0.35 ; p>0.75)$. The total charge in the PP grew with depolarization, especially sharply between -50 and $-20 \mathrm{mV}$ (by $\sim 350 \%, p<0.001$ in paired $t$ test).
These characteristics might be important for the distancedependent integration of synaptic inputs.

\section{DISCUSSION}

The perforant path and Schaffer collateral inputs to CA1 neurons are two well-defined inputs having different sources and terminating on the apical dendrites at different distances from the cell body. We have examined the properties of synaptic transmission at these two inputs and found that they are very different in the properties of the NMDA component and in the ratio of the NMDA to AMPA components. Scaled by the maximal inward current at $-20 \mathrm{mV}$, the outward NMDA-mediated current at $+60 \mathrm{mV}$ in the PP was approximately six times smaller than in the SC (Fig. 2B,C, 3B). This difference was still observed in the absence of extracellular $\mathrm{Mg}^{2+}$. The phenomenon could not be attributed to a failure to detect outward current through distal synapses, because we found that even larger outward AMPAmediated currents in the PP could be detected in the same cell. Furthermore, the fact that the AMPA-mediated currents in the PP have a reversal potential only slightly more positive than expected and have a linear relationship to driving force (Fig. 1) indicates that the ability to manipulate voltage at distal synapses was good. There is thus no reason to suspect that the failure to detect large outward currents in the PP NMDA component can be attributed simply to their distance. We also found that the slow time course of NMDA-mediated EPSCs was not the cause of inward rectification, because large outward currents were observed in the PP when the AMPA currents were prolonged to match the NMDA kinetics.

Because the NMDA channels in the PP were able to carry inward current much better than outward current, we described these channels as being inwardly rectifying. There has been no previous indication that NMDA channels are inwardly rectifying, but this is well known for AMPA channels that lack GluR-B (Verdoorn et al., 1991). Such channels are known to have a much higher $\mathrm{Ca}^{2+}$ permeability than channels with GluR-B, and it would thus be of interest to know whether the inwardly rectifying NMDA channels also have higher $\mathrm{Ca}^{2+}$ permeability. Input selectivity of AMPA transmission in functionally different inputs was demonstrated in CA3 interneurons (Toth and McBain, 1998). In this case, the mossy fiber input contained $\mathrm{Ca}^{2+}$-permeable AMPA receptors, whereas the input from CA1 pyramidal cells did not. It is not yet clear what molecular mechanisms might be responsible for the unusual behavior of the PP NMDA current. One possibility is a more complex composition of the PP NMDA receptors. New data show that NMDA receptor heteromer composition is not limited to an NMDA receptor subunit 1 (NR1)/ NR2 dimeric option. When coexpressed in Xenopus oocytes, 

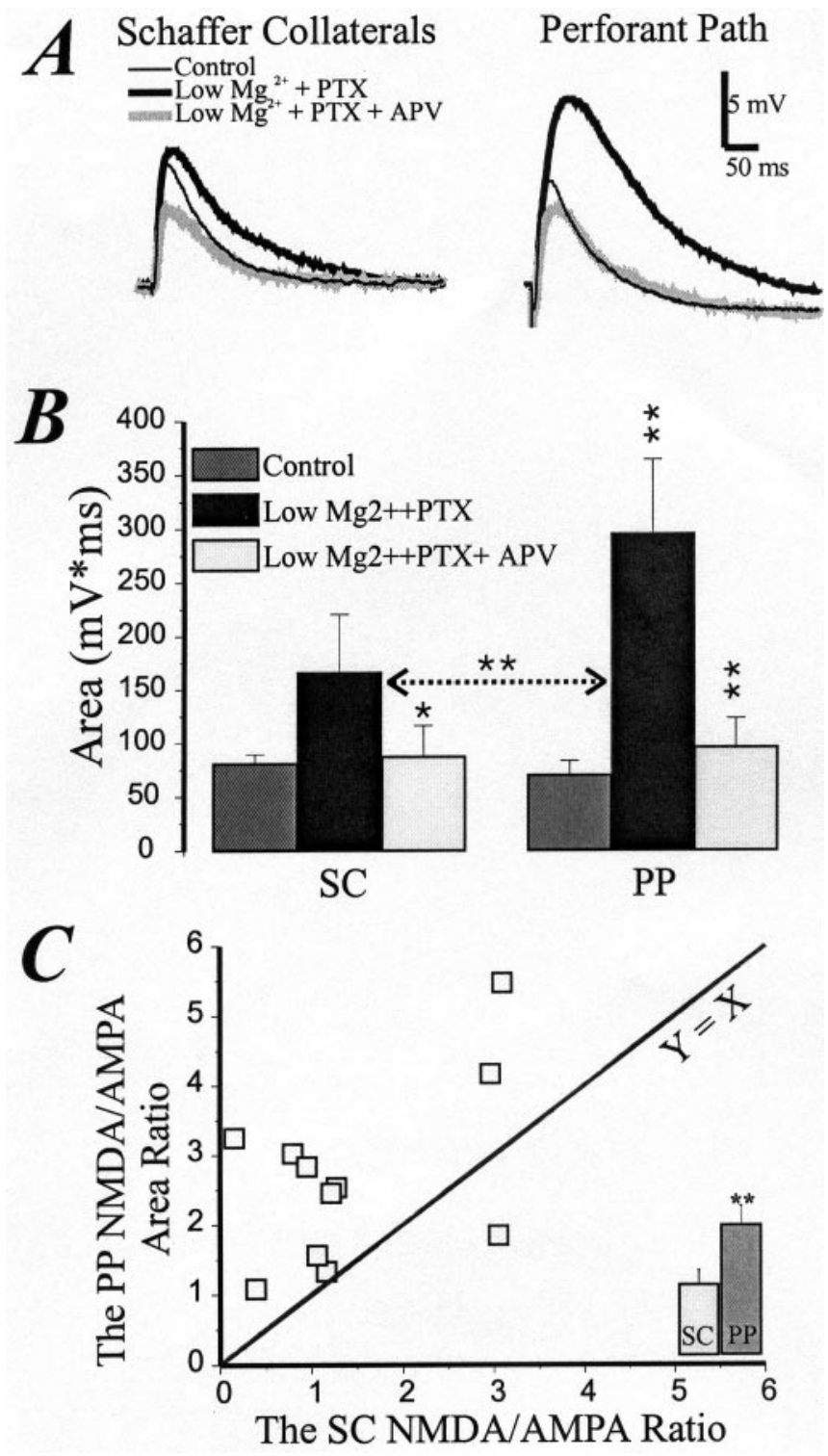

Figure 6. The NMDA/AMPA ratio in ACSF without $\mathrm{Mg}^{2+}$ is higher in the PP than SC input (current-clamp data). $A$, The SC and PP EPSP (average of 10 traces) in control ACSF, in low $\mathrm{Mg}^{2+}$ and picrotoxin, and after the APV application. The scale bar is shown in top right corner. B, Changes in EPSP area that depend on the NMDA component are stronger in the PP than SC input (average of 11 cells). Asterisks indicate the significance of changes compared with previous conditions $\left({ }^{*} p<0.05\right.$; $\left.*^{*} p<0.01\right)$. The horizontal arrow shows the significance of low $\mathrm{Mg}^{2+}+P T X$ and low $\mathrm{Mg}^{2+}+P T X+A P V$ differences between the inputs. $C$, The NMDA/AMPA EPSP area ratio in the PP is higher than in the SC input. Each square represents one cell. ${ }^{* *} p<0.01$. The $Y=X$ function is added for comparison. Inset, The bar graph shows the difference in averaged NMDA/AMPA ratios between the inputs.

NR1, NR2A, and NR2C or NR2D subunits form heteromeric complexes containing all three subunits with new properties not observed in dimeric configurations (Sucher et al., 1996; Cheffings and Colquhoun, 2000). The voltage characteristics of such receptors, however, have not been described. Another possibility is that NMDA receptor-channel properties might be modified by the multimolecular complex surrounding it at the synapse (Sheng and Lee, 2000). Actin-dependent regulation of the NMDA receptor is a well known example of such modification (Rosenmund and
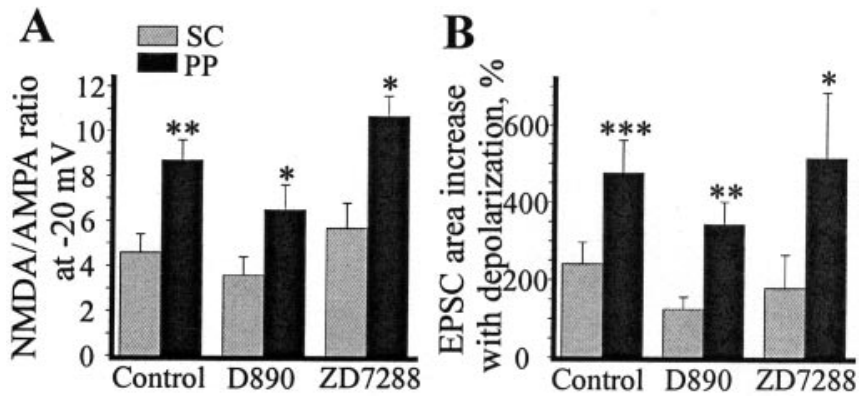

Figure 7. The NMDA/AMPA ratio measured under voltage clamp is consistently higher in PP than in SC input in the presence and in the absence of NMDA blocker. $A$, The NMDA/AMPA ratio measured at $-20 \mathrm{mV}$ (using APV) under three experimental conditions: control $\left(\mathrm{Cs}^{+}\right.$ plus QX-314 inside the cell), D890 $\left(\mathrm{Cs}^{+}\right.$plus QX-314 plus D890 inside the cell), and ZD7288 ( $\mathrm{Cs}^{+}$plus QX-314 inside the cell, ZD7288 in the bath). $B$, Increase in EPSC area (\%) with depolarization from -65 to -20 $\mathrm{mV}$ under three experimental conditions (no APV in ACSF). Asterisks indicate the significance of differences between the two inputs in the paired $t$ test. For $A$ and $B,{ }^{*} p<0.05 ;{ }^{* *} p<0.01 ;{ }^{* *} p<0.001$.

Westbrook, 1993), and it is possible that such complexes might be different in different synaptic pathways.

The second major finding of this study is that the NMDA/ AMPA ratio is different at the two synapses. This ratio was first characterized in voltage clamp under different experimental conditions in regular ACSF. We found that the differences in NMDA/AMPA ratio cannot be attributed to voltage-dependent ion channels. We also controlled for the possibility of a regenerative NMDA-dependent process, measuring the NMDA/AMPA ratio in low- $\mathrm{Mg}^{2+} \mathrm{ACSF}$ using both current-clamp and voltageclamp methods. By both measures, the NMDA/AMPA ratio in the PP was approximately twice as large as in the SC input. This conclusion is consistent with our previous observations using field EPSP (fEPSP) recording in older (32- to 45-d-old) rats (Otmakhova and Lisman, 1999). These showed that in ACSF containing low $\mathrm{Mg}^{2+}$ and picrotoxin, APV suppressed the PP fEPSP by $\sim 40 \%$, twice as strongly as the SC input $(\sim 20 \%)$. The fEPSP method has the advantage of recording close to the PP and SC synaptic sites, eliminating concerns about space-clamp errors.

We believe that the differences we observed were input-specific and not just dependent on the distance of the synapse from the cell body. The NMDA rectification cannot be explained by the distance, because it was always observed in the PP but never in the SC input. The distributions of NMDA/AMPA ratio in the two inputs, however, did partially overlap. We examined whether the distribution in the SC depended on the distance of the stimulating electrode from the cell body and did not find a significant correlation. This finding is consistent with a recent report (Andrasfalvy et al., 2000) showing no signs of a distancedependent gradient of NMDA/AMPA ratios within the stratum radiatum by patching the dendrite at different distances from the cell body. It was shown recently that the stratum lacunosum moleculare has significantly larger synapses, with a higher percentage of the perforated type compared with the stratum radiatum, and that $\sim 30 \%$ of these synapses are directly positioned on dendrites, not on spines (Megias et al., 2001). We found no published immunohistological data, however, on the NMDA/ AMPA composition of the PP and SC synapses.

The evidence presented here is the first to show that CA1 pathways with different proximal/distal input locations can have different properties of the NMDA component. A related pathway 
effect was noted for the CA3 region, where the proximal mossy fiber input had a smaller NMDA component than more distal commissural-associational input (Williams and Johnston, 1991; Jonas et al., 1993; Watanabe et al., 1998). Interestingly, the NMDA spike was also observed in most distal branches of basal dendrites of cortical pyramidal cells (Schiller et al., 2000). These findings suggest the possibility of a general rule by which more distant inputs have a higher NMDA/AMPA ratio.

We have identified properties of the PP input relevant to the model of Cook and Johnston (1999). According to this model, one of the conditions for eliminating the location dependence of synaptic inputs is voltage independence of the synaptic current (a synapse acting as a perfect current source). We found that the peak amplitude of the PP EPSC did not change between -70 and $-40 \mathrm{mV}$, whereas the SC amplitude progressively decreased. Therefore, below $-40 \mathrm{mV}$, the PP input may act as a perfect current source. The SC input showed a different but potentially important property: when total charge was measured, it was constant at voltages between -70 and $-40 \mathrm{mV}$. It is conceivable that different neural computations can effectively use these invariances.

The two CA1 pathways we have studied have different functional roles (for review, see Lisman and Otmakhova, 2001) and are differentially controlled by neuromodulators (Lee et al., 1983; Hasselmo and Schnell, 1994; Otmakhova and Lisman, 1999, 2000). The understanding of their interaction under different conditions is necessary for understanding the hippocampal function in general. Our results represent a step in this direction, because the pathway-specific differences in the voltage-dependent NMDA component could have important consequences for the interaction of these pathways.

\section{REFERENCES}

Andrasfalvy B, Teppre J, Magee JC (2000) Characterization of synaptic glutamate receptors in the dendrites of adult rat CA1 pyramidal cells. Soc Neurosci Abstr 26:421.10.

Andreasen M, Lambert JD (1999) Somatic amplification of distally generated subthreshold EPSPs in rat hippocampal pyramidal neurones. J Physiol (Lond) 519:85-100.

Bernander O, Douglas RJ, Martin KA, Koch C (1991) Synaptic background activity influences spatiotemporal integration in single pyramidal cells. Proc Natl Acad Sci USA 88:11569-11573.

Bragin AG, Otmakhov NA (1979) Comparison of direct influences of the perforant path on hippocampal CA1 and CA3 neurons in vitro. Neurophysiology 11:220-225.

Calton JL, Kang MH, Wilson WA, Moore SD (2000) NMDA-receptordependent synaptic activation of voltage-dependent calcium channels in basolateral amygdala. J Neurophysiol 83:685-692.

Carnevale NT, Johnston D (1982) Electrophysiological characterization of remote chemical synapses. J Neurophysiol 47:606-621.

Cheffings CM, Colquhoun D (2000) Single channel analysis of a novel NMDA channel from Xenopus oocytes expressing recombinant NR1a, NR2A and NR2D subunits. J Physiol (Lond) 526:481-491.

Connors BW, Prince DA (1982) Effects of local anesthetic QX-314 on the membrane properties of hippocampal pyramidal neurons. J Pharmacol Exp Ther 220:476-481.

Cook EP, Johnston D (1999) Voltage-dependent properties of dendrites that eliminate location-dependent variability of synaptic input. J Neurophysiol 81:535-543.

Doller HJ, Weight FF (1982) Perforant pathway activation of hippocampal CA1 stratum pyramidale neurons: electrophysiological evidence for a direct pathway. Brain Res 237:1-13.

Golding NL, Spruston N (1998) Dendritic sodium spikes are variable triggers of axonal action potentials in hippocampal CA1 pyramidal neurons. Neuron 21:1189-1200.

Hasselmo ME, Schnell E (1994) Laminar selectivity of the cholinergic suppression of synaptic transmission in rat hippocampal region CA1: computational modeling and brain slice physiology. J Neurosci $14: 3898-3914$

Hausser M, Stuart GJ (2001) Dendritic coincidence detection of EPSPs and action potentials. Nat Neurosci 4:63-71.
Hausser M, Major G, Stuart GJ (2001) Differential shunting of EPSPs by action potentials. Science 291:138-141.

Hestrin S, Nicoll RA, Perkel DJ, Sah P (1990) Analysis of excitatory synaptic action in pyramidal cells using whole-cell recording from rat hippocampal slices. J Physiol (Lond) 422:203-225.

Hoffman DA, Magee JC, Colbert CM, Johnston D (1997) $\mathrm{K}^{+}$channel regulation of signal propagation in dendrites of hippocampal pyramida neurons. Nature 387:869-875.

Johnson RR, Jiang X, Burkhalter A (1996) Regional and laminar differences in synaptic localization of NMDA receptor subunit NR1 splice variants in rat visual cortex and hippocampus. J Comp Neurol 368:335-355.

Jonas P, Major G, Sakmann B (1993) Quantal components of unitary EPSCs at the mossy fibre synapse on CA3 pyramidal cells of rat hippocampus. J Physiol (Lond) 472:615-663.

Kovalchuk Y, Eilers J, Lisman J, Konnerth A (2000) NMDA receptormediated subthreshold $\mathrm{Ca}^{2+}$ signals in spines of hippocampal neurons J Neurosci 20:1791-1799.

Lambert NA, Wilson WA (1993) Discrimination of post- and presynaptic $\mathrm{GABA}_{\mathrm{B}}$ receptor-mediated responses by tetrahydroaminoacridine in area CA3 of the rat hippocampus. J Neurophysiol 69:630-635.

Lee KS, Schubert P, Reddington M, Kreutzberg GW (1983) Adenosine receptor density and the depression of evoked neuronal activity in the rat hippocampus in vitro. Neurosci Lett 37:81-85.

Lisman JE, Otmakhova NA (2001) Storage, recall, and novelty detection of the sequences by the hippocampus: elaborating the SOCRATIC model to account for normal, aberrant effects of dopamine. Hippocampus 11:551-568.

Magee J (1999) Dendritic $I_{\mathrm{h}}$ normalizes temporal summation in hippocampal CA1 neurons. Nat Neurosci 2:508-514.

Magee JC (1998) Dendritic hyperpolarization-activated currents modify the integrative properties of hippocampal CA1 pyramidal neurons. J Neurosci 18:7613-7624.

Magee JC, Cook EP (2000) Somatic EPSP amplitude is independent of synapse location in hippocampal pyramidal neurons. Nat Neurosci 3:895-903.

Major G (1993) Solutions for transients in arbitrarily branching cables III. Voltage clamp problems. Biophys J 65:469-491.

Major G, Evans JD, Jack JJ (1993a) Solutions for transients in arbitrarily branching cables. I. Voltage recording with a somatic shunt. Biophys J 65:423-449.

Major G, Evans JD, Jack JJ (1993b) Solutions for transients in arbitrarily branching cables. II. Voltage clamp theory. Biophys J 65:450-468.

McNaughton BL, Barnes CA, Meltzer J, Sutherland RJ (1989) Hippocampal granule cells are necessary for normal spatial learning but not for spatially-selective pyramidal cell discharge. Exp Brain Res 76:485-496.

Megias M, Emri Z, Freund TF, Gulyas AI (2001) Total number and distribution of inhibitory and excitatory synapses on hippocampal CA1 pyramidal cells. Neuroscience 102:527-540.

Mlinar B, Pugliese A, Corradetti R (2001) Selective inhibition of local excitatory synaptic transmission by serotonin through an unconventional receptor in the CA1 region of rat hippocampus. J Physiol (Lond) 534:141-158.

Otmakhova NA, Lisman JE (1999) Dopamine selectively inhibits the direct cortical pathway to the CA1 hippocampal region. J Neurosci 19:1437-1445

Otmakhova NA, Lisman JE (2000) Dopamine, serotonin, noradrenaline strongly inhibit the direct perforant path-CA1 input, but have little effect on the Schaffer collateral input. In: Parahippocampal region: implications for neurological and psychiatric diseases (Schafman HE Witter MP, Schwarcz R, eds), pp 462-464. New York: New York Academy of Sciences.

Otmakhova NA, Otmakhov N, Mortenson LH, Lisman JE (2000) Inhibition of the CAMP pathway decreases early long-term potentiation at CA1 hippocampal synapses. J Neurosci 20:4446-4451.

Perkins KL, Wong RK (1995) Intracellular QX-314 blocks the hyperpolarization-activated inward current $I_{\mathrm{q}}$ in hippocampal CA1 pyramidal cells. J Neurophysiol 73:911-915.

Rosenmund C, Westbrook GL (1993) Calcium-induced actin depolymerization reduces NMDA channel activity. Neuron 10:805-814.

Schiller J, Major G, Koester HJ, Schiller Y (2000) NMDA spikes in basal dendrites of cortical pyramidal neurons. Nature 404:285-289.

Seamans JK, Gorelova NA, Yang CR (1997) Contributions of voltagegated $\mathrm{Ca}^{2+}$ channels in the proximal versus distal dendrites to synaptic integration in prefrontal cortical neurons. J Neurosci 17:5936-5948.

Sheng M, Lee SH (2000) Growth of the NMDA receptor industrial complex. Nat Neurosci 3:633-635.

Spruston N, Jaffe DB, Williams SH, Johnston D (1993) Voltage- and space-clamp errors associated with the measurement of electrotonically remote synaptic events. J Neurophysiol 70:781-802.

Spruston N, Jonas P, Sakmann B (1995) Dendritic glutamate receptor channels in rat hippocampal CA3 and CA1 pyramidal neurons. J Physiol (Lond) 482:325-352.

Stuart G, Sakmann B (1995) Amplification of EPSPs by axosomatic 
sodium channels in neocortical pyramidal neurons. Neuron 15:1065-1076.

Sucher NJ, Awobuluyi M, Choi YB, Lipton SA (1996) NMDA receptors: from genes to channels. Trends Pharmacol Sci 17:348-355.

Talbot MJ, Sayer RJ (1996) Intracellular QX-314 inhibits calcium currents in hippocampal CA1 pyramidal neurons. J Neurophysiol $76: 2120-2124$.

Toth K, McBain CJ (1998) Afferent-specific innervation of two distinct AMPA receptor subtypes on single hippocampal interneurons. Nat Neurosci 1:572-578.

Verdoorn TA, Burnashev N, Monyer H, Seeburg PH, Sakmann B (1991) Structural determinants of ion flow through recombinant glutamate receptor channels. Science 252:1715-1718.

Vinogradova O (1984) Functional organization of the limbic system in the process of registration of information: facts and hypotheses. In: The hippocampus (Isaacson RL, Pribram KH, eds), pp 1-69. New York: Plenum.

Watanabe M, Fukaya M, Sakimura K, Manabe T, Mishina M, Inoue Y (1998) Selective scarcity of NMDA receptor channel subunits in the stratum lucidum (mossy fibre-recipient layer) of the mouse hippocampal CA3 subfield. Eur J Neurosci 10:478-487.

Williams SH, Johnston D (1991) Kinetic properties of two anatomically distinct excitatory synapses in hippocampal CA3 pyramidal neurons. J Neurophysiol 66:1010-1020.

Yeckel MF, Berger TW (1990) Feedforward excitation of the hippocampus by afferents from the entorhinal cortex: redefinition of the role of the trisynaptic pathway. Proc Natl Acad Sci USA 87:5832-5836.

Yoon KW, Wotring VE, Fuse T (1998) Multiple picrotoxinin effect on glycine channels in rat hippocampal neurons. Neuroscience 87:807-815. 